Background/Purpose Pathogenic antibody-secreting cells (ASC) are poorly characterized in human lupus nephritis (LN). Our objective was to compare the single cell molecular signature of ASC in kidney and urine from patients with active LN, either untreated or after immunosuppressive therapy failure.

Methods ASC were identified by anti-CD138 staining on fixed renal biopsies from patients with active LN. We sorted singleASC from fresh renal biopsies to perform gene expression profiling. ASC transcriptional program from urine of untreated LN patients was assessed at diagnosis and after a prospective follow up during induction therapy.

Results Interstitial infiltrates of CD138+ ASC were found in untreated $(\mathrm{N}=15)$ and refractory patients $(\mathrm{N}=6)$. Single cell molecular signature of kidney ASC from untreated patients revealed that these cells were mostly plasmablasts and contrasted with ASC signature from patients with mycophenolate mofetil failure that expressed long-lived plasma cells genes and clustered with long-lived bone marrow ASC from healthy donors. A plasmablast signature was observed in urine ASC at diagnosis, similar to their kidney counterpart. The concentration of urine ASC from 22 untreated patients correlated with ISN/RPS classification, with higher concentration in class IV patients $(\mathrm{p}<0.01)$.

Conclusion These results suggest that plasmablasts infiltrate kidney of untreated LN patients, while kidney long-lived ASC may contribute to the failure of immunosuppressive therapy. Acknowledgement This work was funded by FOREUM.

\section{O20 MARGINAL ZONE B CELL DEVELOPMENT FROM EARLY T2 PROGENITORS IS DEFECTIVE IN LUPUS NEPHRITIS}

${ }^{1}$ Thomas J Tull, 'William Guesdon, ${ }^{2}$ Michael G Robson, 'David D'Cruz, ${ }^{3}$ Mats Bemark, ${ }^{1}$ Jo Spencer. 'School of Immunology and Microbial Sciences, King's College London, London; 'Dept. of Nephrology, Guy's and St Thomas' NHS Trust, London, UK; ${ }^{3}$ Dept. of Microbiology and Immunology, University of Gothenburg, Gothenburg, Sweden

10.1136/lupus-2020-eurolupus.31

Background Marginal zone B cells (MZB) are a distinct B cell subset but the site and nature of their differentiation in humans is not understood. In mice, MZB are formed from transitional T2 cells shortly after exit from the bone marrow into the blood. Here we demonstrate a human MZB maturation axis that emerges at the T2 stage that collapses in lupus nephritis (LN).

Methods Mass cytometry was used to phenotype B cells from blood and gut-associated lymphoid tissue (GALT) from healthy donors (HCD). Single cell RNA sequencing was performed using a 10X genomics platform. Flow and mass cytometry were used to investigate MZB differentiation in LN.

Results Deep phenotypic analysis of B cells from HCD revealed prominent $\operatorname{IgM}^{\text {hi }}$ and $\operatorname{IgM}^{\text {lo }}$ TS cell populations emerge at the T2 stage and phenotypically align with $\mathrm{IgM}^{\mathrm{hi}} \mathrm{CD} 45 \mathrm{RB}^{\mathrm{hi}}$ marginal zone precursor cells (MZP). IgM ${ }^{\text {hi }}$ TS cells had high expression of the gut homing receptor $\alpha 4 \beta 7$ integrin and were grossly enriched in GALT. IgM ${ }^{\text {hi }}$ TS $B$ cells produced IL-10 and were enriched in retinoic acid and lipopolysaccharide genes consistent with exposure to the gut microenvironment. MZB depletion was seen in $\mathrm{LN}$ alongside depletion of MZP and TS $\operatorname{IgM}^{\text {hi }}$ cells, suggesting collapse of this maturational axis.

Conclusion We identify a bifurcation of human B cell development that starts at the T2 stage and gives rise to a gut homing $\operatorname{IgM}^{\mathrm{hi}}$ branch that is functionally and phenotypically distinct. The depletion of this branch in patients with LN affirms its existence in health, but also has important disease implications, such as the susceptibility of LN patients to pneumococcal infections and diminished $\mathrm{B}$ regulatory responses seen in the disease.

Acknowledgements This work was funded by the Medical Research Council of Great Britain (MR/R000964/1) and The Lupus Trust.

\section{ANALYSIS OF B-CELL INFILTRATES AND TERTIARY LYMPHOID ORGAN IN LUPUS NEPHRITIS}

Fahd Touzani, Agnieszka Pozdzik. Nephrology Dept., Brugmann Hospital, Brussels, Belgium

\subsection{6/lupus-2020-eurolupus.32}

Background Renal impairment is the leading cause of morbidity and mortality in systemic lupus erythematosus. Tertiary lymphoid organs (TLO) are organized lymphoid structures that develop in response to inflammatory signals from tissues. They may organize into a functional ectopic germinal center. Their pathogenic role in the evolution of kidney function in lupus nephritis remains uncertain.

Method To assess the correlation between tertiary lymphoid organs and B-cell infiltrates and the severity and outcome of kidney function, we conducted a retrospective study of renal biopsy in patients with lupus nephritis. Immunophenotyping of the cell infiltrate was evaluated by immunohistochemistry. We assessed the B-cell infiltrate with a semi-quantitative score. Results 18 adult patient biopsies identified over the 10 -year period met the inclusion criteria.

Immunophenotyping of the inflammatory infiltrate was performed in 17 patients. There was no inflammatory infiltrate in 3 patients. Scattered $\mathrm{T}$ and $\mathrm{B}$ cells were found in 4 patients and $40 \%$ of patients showed organized clusters of $\mathrm{T}$ and $\mathrm{B}$ lymphocytes (grade 3 and 4 infiltration) with TLO in 2 biopsies. 9 of the 18 patients went into complete renal remission. Of the 12 patients with unorganized inflammatory infiltrates (grade $0,1,2), 7$ patients $(58.3 \%)$ achieved recovery. In contrast, 2 out of 5 patients $(40 \%)$ with organized infiltrates (3 and 4$)$ reached complete renal remission $(p=0.44)$. The time to renal remission was longer in patient with unorganized infiltration than those with organized infiltrate (median time over 24 vs. 18.9 months respectively, $\mathrm{p}=0.24$ ) (figure 1). Of

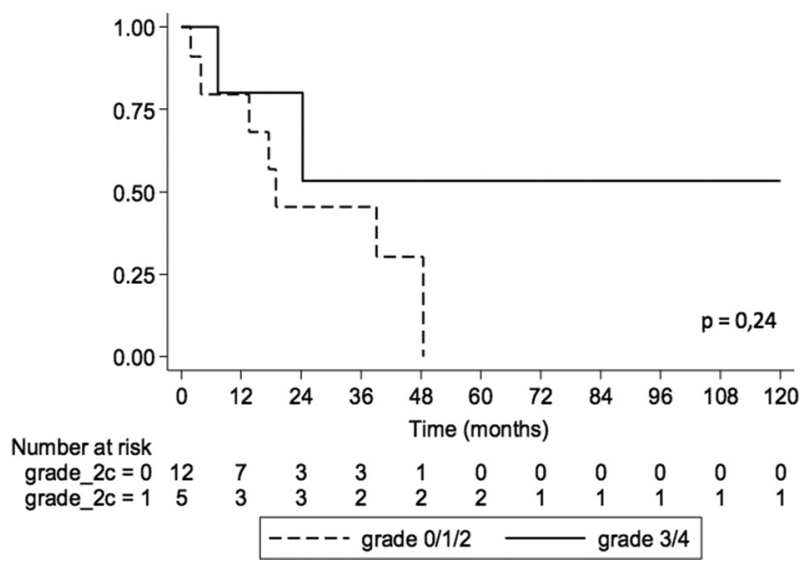

Abstract 021 Figure 1 Kaplan Meier curve of renal remission according to the inflammatory infiltrate 
the 9 patients with renal remission, 4 relapsed. Finally, 4 patients required dialysis, including 1 death.

Conclusion The presence of intrarenal B cells rarely forms TLO in lupus nephritis. Due to the small sample size, we were unable to determine their prognostic role. Nevertheless, we report here the longer time to renal remission for grade 3 and 4 infiltrates; prospective studies with repeated renal biopsies are needed to better characterize their relationship to disease progression.

Funding None

\section{O22 CORRELATION BETWEEN INTERSTITIAL CD8+ T CELL INFILTRATION AND FIBROTIC PROCESSES IN A MOUSE MODEL OF LUPUS NEPHRITIS}

1,2 Pauline Montigny, ${ }^{1}$ Aurélie Degroof, ${ }^{3}$ Davide Brusa, ${ }^{1,4}$ Frédéric Houssiau,
${ }^{1,4}$ Bernard Lauwerys. ${ }^{1}$ Pôle de Pathologies Rhumatismales Systémiques et Inflammatoires,
UCLouvain, Louvain; ${ }^{2}$ Service de Rhumatologie, CHU UCL Namur, Yvoir; ${ }^{3}$ Plateforme de
Cytométrie de Flux, Institut de Recherche Expérimentale et Clinique, UCLouvain, Louvain;
${ }^{4}$ Service de Rhumatologie, Cliniques Universitaires Saint Luc, Bruxelles, Belgium

10.1136/lupus-2020-eurolupus.33

Background Tubulo-interstitial damage during lupus nephritis $(\mathrm{LN})$ is associated with poor renal prognosis in the long run. Here, we describe the progression of tubulo-interstitial fibrosis and immune cells infiltration with emphasis on CD8 + T cells, in parallel with renal outcomes in a mouse lupus model.

Methods We collected blood, urine and kidneys from 39 B6/ Sle1.Sle2.Sle3 lupus-prone mice, before disease onset and at different stages of disease progression. RNA was extracted from kidneys and hybridized on Mouse Gene 2.0 ST exon arrays. Histopathological scores (NIH Activity and Chronicity Indexes) and digital quantification of fibrosis, IgGK deposits, $\mathrm{CD} 8+$ and $\mathrm{CD} 3+\mathrm{T}$ cells were performed on total kidney. Renal CD8 $+\mathrm{T}$ cell phenotypes were determined by flow cytometry. Plasma urea and albuminuria were measured by immunoenzymatic assays.

Results IgGк deposits, CD8 $+\mathrm{T}$ cell infiltration and interstitial fibrosis increase with the progression of renal disease, evaluated by histopathological scores and plasma urea. Further, digital quantifications allowed us to identify a significant correlation $(\mathrm{r}=0,45, p=0,011)$ between local CD8 $+\mathrm{T}$ cell population and fibrosis, while total $\mathrm{CD} 3+$ cells population and

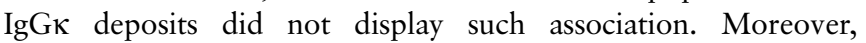
characterization of $\mathrm{CD} 8+\mathrm{T}$ cell subpopulations showed that fibrosis is more specifically linked to effector functions of
CD8 $+\mathrm{T}$ cells. Transcriptomic analyses supported this association, with a high correlation coefficient between mean expression of effector functions transcripts and the presence of a fibrotic signature $(r=0,92, p<0,0001)$.

Conclusions Our results support the association between CD8 $+\mathrm{T}$ cell tubulo-interstitial infiltration and renal outcomes in a mouse lupus model. Further, a strong correlation is identified between effector functions of CD8 $+\mathrm{T}$ cells and fibrotic processes, opening new avenues of research in the pathogenesis of LN.

Acknowledgements This work was supported by F.R.S-F.N.R.S and by Fondation Roi Baudouin

\section{O23 IDENTIFICATION OF PROTEIN-QUANTITATIVE TRAIT LOCI (PQTLS) IN THE INTERFERON SIGNALLING PATHWAY}

Christian Lundtoft, Pascal Pucholt, Johanna K Sandling, Lars Rönnblom, Niklas Hagberg. Dept. of Medical Sciences, Rheumatology, Uppsala University, Uppsala, Sweden

\subsection{6/lupus-2020-eurolupus.34}

Background Interferon (IFN)- $\alpha$ and IFN- $\gamma$ are important cytokines in the pathogenesis of systemic lupus erythematosus (SLE), and several of the genetic associations with SLE are found in genes that are fundamental for the IFN response (e. g. TYK2, STAT4, IRF5). This study aimed to define the genetic regulation of the IFN system, and to link disease-associated SNPs to alterations in the IFN system.

Methods Peripheral blood mononuclear cells from 303 healthy individuals were stimulated with IFN- $\alpha$ or IFN- $\gamma$ (figure 1). Basal levels of IFN-receptors (IFNAR2 and IFNGR1) and IFN-induced phosphorylation of STAT1 and STAT4, expression of CXCL9, CXCL10, HLA-ABC and HLA-DRPQ was determined in 6 cell subsets using flow cytometry. Each readout was mapped as a pQTL using 3.4 million SNPs with a minor allele frequency $\geq 5 \%$ (Illumina Global Screening Array with subsequent genome-wide imputation) in an additive model correcting for covariates. pQTLs were probed for overlap with GWAS SLE-associated SNPs.

Results We identified 8 genome-wide significant pQTLs $(\mathrm{p}<5.0 \mathrm{e}-8), 3$ of which were associated with basal IFN receptor levels, 3 with IFN- $\alpha$, and 2 with IFN- $\gamma$-induced traits. One pQTL affected protein expression in cis (IFNAR2), whereas the other were trans-pQTLs. The strongest association was observed for a SNP in HLA-A that affected IFNAR2 level

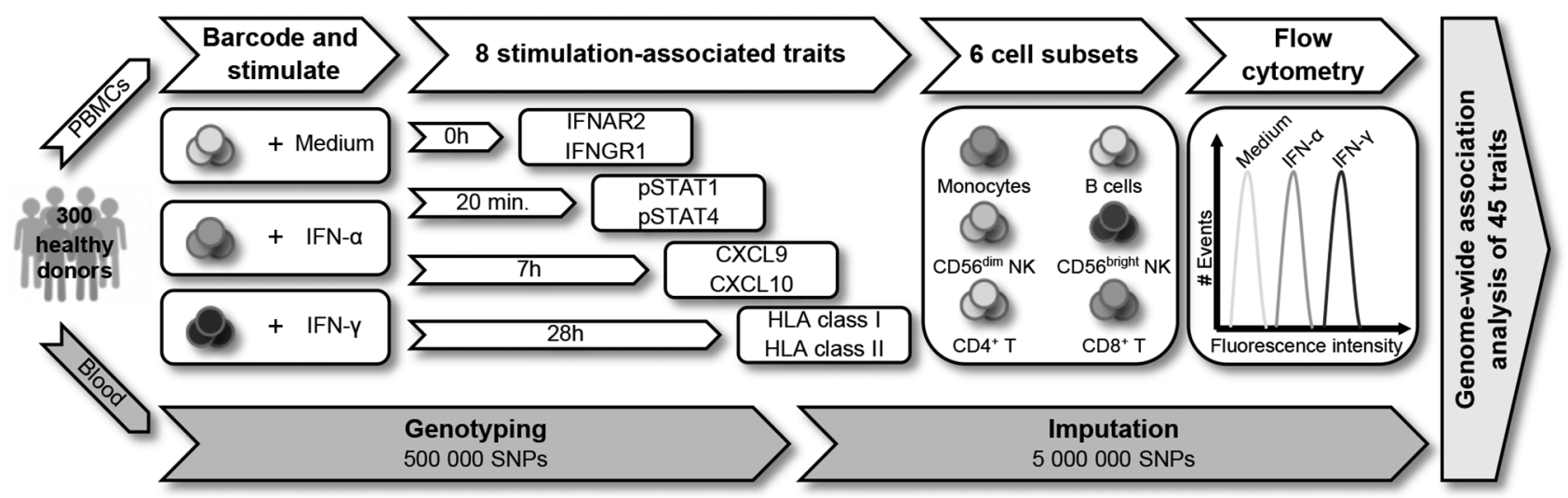

Abstract 023 Figure 1 Graphical study design 